\title{
AN ASYMPTOTIC BOUND \\ FOR THE ITERATES OF CERTAIN REAL FUNCTIONS NEAR A CONTRACTIVE FIXED POINT
}

\author{
LAWRENCE J. WALLEN
}

(Communicated by R. Daniel Mauldin)

\begin{abstract}
If $x=0$ is a contractive fixed point for the function $F$, then under certain conditions, the iterates $F_{k}(a)$ are asymptotically equal to the numbers $\xi_{k}$ defined by $k=\int_{\xi_{k}}^{a} \frac{d u}{u-F(u)}$. Using somewhat different hypotheses, we give a more precise bound on $F_{k}(a) / \xi_{k}$.
\end{abstract}

Let $F$ be a real function of the real variable $x$, defined and continuous for $0 \leq x \leq A$, with $0<F(x)<x$ for $0<x \leq A$. The $k$ th iterate of $F$ will be denoted by $F_{k}\left(F_{0}(x) \equiv x\right)$. Set the error $x-F(x)=G(x)$. In the classical case where $G(x) \sim C x^{\alpha}, C>0, \alpha>1$, the asymptotic formula $F_{k}(a) \sim((\alpha-1) C k)^{1 / 1-\alpha}$ has been known for a long time (see [1] or [3]).

In the elementary note [2], the author and V. Drobot dealt with certain other cases and proved that when $F$ converges to 0 "slowly" and certain other conditions are met, then the first asymptotic term is given by $\xi_{k}$ where $\xi_{k}$ is defined by the equation

$$
k=\int_{\xi_{k}}^{a} \frac{d u}{G(u)} .
$$

In addition, we gave several heuristic arguments for $(*)$. The theorem proved in [2] follows.

Theorem 0. With the notation given previously, assume $G \in C^{1}, G(0)=G^{\prime}(0)=$ 0 , and $G$ is strictly increasing. Define

$$
H(x)=\int_{x}^{a} \frac{d u}{G(u)}
$$

so that $H\left(\xi_{k}\right)=k$. Assume that for each $\mu>1$,

$$
\limsup _{x \rightarrow 0} \frac{H(\mu x)}{H(x)}<1 \text {. }
$$

Then $\xi_{k} \sim F_{k}(a)$.

Received by the editors July 10, 1989.

1980 Mathematics Subject Classification (1985 Revision). Primary 26A18. 
The main purpose of this note is to give a bound for $F_{k}(a) / \xi_{k}$. We do this by imposing a new criterion for "slow convergence to 0 ." Moreover, under this condition, we can dispense with the unwanted assumption that $G^{\prime}(0)=0$. In fact, the result will apply to certain functions composed of line segments whose slopes take successively the values 0 and 1 . Although, in the generality of our setting, we cannot get a true second asymptotic term, our error has the correct order of magnitude in the classical case.

Theorem 1. With the notation from above, assume $F$ and $G$ are increasing for $0 \leq x \leq A$. Let $K$ denote the function inverse to $H$, and set $Q=-K^{\prime}$. Assume moreover that

$$
\lim _{\theta \downarrow 1} \limsup _{x \rightarrow \infty} Q(x) / Q(\theta x)=1 .
$$

Then there is a positive constant $C$, so that if $k \geq 1$ and $\xi_{\ell+1} \leq F_{k}(a)<\xi_{\ell}$, then $\ell \geq k>\ell-C \log \ell$ and $\xi_{k} \sim F_{k}(a)$. Moreover, $C$ can be chosen independent of a for $a \leq a_{0}$.

Remarks. The hypothesis on $Q$ is equivalent to the condition that $\log Q$ be slowly oscillating. The conditions imposed on $F$ and $G$ trivially imply that for $0<x<y<A, 0<F(y)-F(x)<y-x$. Since $0<G(x)<x$ and is increasing, $K$ is defined for $0 \leq x<\infty$ and is convex. Thus $Q=-K^{\prime}=G(K)$. We denote $F_{k}(a)$ by $\eta_{k}$.

Proof of Theorem 1. First, note that $F\left(\xi_{\ell}\right)<\xi_{\ell+1}$, for $\xi_{\ell+1}-F\left(\xi_{\ell}\right)=\xi_{\ell+1}-$ $\xi_{\ell}+G\left(\xi_{\ell}\right)=K(\ell+1)-K(\ell)-K^{\prime}(\ell)>0$ by the convexity of $K$. If $\eta_{j}<\xi_{\ell}$, then $\eta_{j+1}=F\left(\eta_{j}\right)<F\left(\xi_{\ell}\right)<\xi_{\ell+1}$, so that every interval $\left[\xi_{\ell+1}, \xi_{\ell}\right)$ contains at most one $\eta$.

Now suppose $\eta_{k+1}<\xi_{\ell+1}<\xi_{\ell} \leq \eta_{k}$. We will prove inductively that for $n>0$,

$$
0<\xi_{\ell+n}-\eta_{k+n} \leq K(\ell+n)-K(\ell)-\sum_{j=0}^{n-1} K^{\prime}(\ell+j)
$$

with strict inequality for $n>1$.

To start the induction we have $F\left(\xi_{\ell}\right) \leq \eta_{k+1}<\xi_{\ell+1}$, so $0<\xi_{\ell+1}-\eta_{k+1} \leq$ $\xi_{\ell+1}-F\left(\xi_{\ell}\right)=K(\ell+1)-K(\ell)-K^{\prime}(\ell)$. Assuming (**) for $n$, we have $\eta_{k+n}<\xi_{\ell+n}$ and so $\eta_{k+n+1}<F\left(\xi_{\ell+n}\right)<\xi_{\ell+n+1}$. Using the Lipschitz condition satisfied by $F$ and the induction hypothesis, we get

$$
\begin{aligned}
0<\xi_{\ell+n+1}-\eta_{k+n+1}= & \xi_{\ell+n+1}-F\left(\xi_{\ell+n}\right)+F\left(\xi_{\ell+n}\right)-F\left(\eta_{k+n}\right) \\
< & \xi_{\ell+n+1}-\xi_{\ell+n}+G\left(\xi_{\ell+n}\right)+\left(\xi_{\ell+n}-\eta_{k+n}\right) \\
\leq & K(\ell+n+1)-K(\ell+n)-K^{\prime}(\ell+n) \\
& +\left(K(\ell+n)-K(\ell)-\sum_{j=0}^{n-1} K^{\prime}(\ell+j)\right) \\
& =K(\ell+n+1)-K(\ell)-\sum_{j=0}^{n} K^{\prime}(\ell+j),
\end{aligned}
$$

so $(* *)$ is established. 
For how many $n$ is it true that $\xi_{\ell+n}-\eta_{k+n}<\xi_{\ell+n}-\xi_{\ell+n+1}$ ? By (**), this condition will hold providing that

$$
K(\ell+n)-K(\ell)-\sum_{j=0}^{n-1} K^{\prime}(\ell+j)<K(\ell+n)-K(\ell+n+1),
$$

or, equivalently,

$$
\sum_{j=0}^{n-1} Q(\ell+j)<\int_{\ell}^{\ell+n+1} Q(u) d u .
$$

Since $Q$ is decreasing, it is enough to have

$$
\sum_{j=0}^{n-1} Q(\ell+j)<\sum_{j=1}^{n+1} Q(\ell+j)
$$

or $Q(\ell)<Q(\ell+n)+Q(\ell+n+1)$ and so $Q(\ell)<2 Q(\ell+n+1)$ suffices. Now if $\theta_{0}>1$ and is sufficiently small, $Q(x) / Q\left(\theta_{0} x\right)<2$, provided $x$ is large. Picking $1<\theta<\theta_{0}$ and a suitably large index $\ell^{\prime}$, we obtain the fact that if $\ell+n<\theta \ell, \ell>\ell^{\prime}$, then we do indeed have $\xi_{\ell+n}-\eta_{k+n}<\xi_{\ell+n}-\xi_{\ell+n+1}$. To recapitulate, if an interval $\left[\xi_{\ell+1}, \xi_{\ell}\right)$ contains no $\eta$ and $\ell$ is large, then the intervals $\left[\xi_{\ell+j+1}, \xi_{\ell+j}\right)$ contain exactly one $\eta$ for $\ell+j<\theta \ell$.

We may assume that there are infinitely many intervals $\left[\xi_{\ell+1}, \xi_{\ell}\right)$ not containing an $\eta$. Denote by $\ell_{\nu}, \nu \geq 0, \ell_{\nu}<\ell_{\nu+1}$, those indices for which $\left[\xi_{\ell+1}, \xi_{\ell}\right)$ has no $\eta$ and $\ell_{0}>\ell^{\prime}$. By the results found earlier, we have $\ell_{1} \geq \theta \ell_{0}$ and, in general, $\ell_{\nu} \geq \theta^{\nu} \ell_{0}$. Of course, since $\eta_{0}=\xi_{0}$, we have $\eta_{k}<\xi_{k}$, so $\ell \geq k$. Assume first that $k \geq \ell_{0}$, and let $\xi_{\ell+1} \leq \eta_{k}<\xi_{\ell}$. Define $\mu$ by $\ell_{\mu+1}>\ell>\ell_{\mu}$. Then $\ell=k+\mu+1+B$ where $B$ is the number of intervals $\left[\xi_{j+1}, \xi_{j}\right), 1 \leq j \leq \ell^{\prime}$, which contain no $\eta$. Hence we have $\log \ell>\log \ell_{\mu}>\mu \log \theta$, and $k>\ell-(\log \theta)^{-1} \log \ell-\left(\ell^{\prime}+1\right)$. By considering separately the case $\ell^{\prime} \leq k<\ell_{0}$ for which $k=\ell-B \geq \ell-\ell^{\prime}$ and the case $k \leq \ell^{\prime}$, we easily obtain the first assertion of the theorem.

To check the uniformity of $C$, let $b<a . H_{b}(x)$ is defined by $H_{b}(x)=$ $\int_{x}^{b} \frac{d u}{G(u)}$, and $K_{b}$ and $Q_{b}$ are defined in the obvious way. For $x>0, Q_{b}(x)=$ $Q(x+H(b))$ and

$$
\frac{Q_{b}(x)}{Q_{b}\left(\theta_{0} x\right)}=\frac{Q(x+H(b))}{Q\left(\theta_{0} x+H(b)\right)}<\frac{Q(x+H(b))}{Q\left(\theta_{0}(x+H(b))\right.}<2
$$

if $x+H(b)>\ell^{\prime}$. Since $C$ is determined by $\theta_{0}$ and $\ell^{\prime}$, uniformity is proved.

Finally, to check that $\xi_{k} \sim \eta_{k}$, note that $K$ satisfies the same slowly oscillating condition as $Q$. If $\varepsilon$ is small and $\theta>1$ is suitably chosen, we get from $K(x)=\int_{x}^{\infty} Q(u) d u$ the inequalities

$$
K(x)<(1+\varepsilon) \int_{x}^{\infty} Q(\theta u) d u=\frac{(1+\varepsilon)}{\theta} \int_{\theta x}^{\infty} Q(u) d u<(1+\varepsilon) K(\theta x)
$$


provided $x$ is large. Now if $\xi_{\ell+1} \leq \eta_{k}<\xi_{\ell}$, then for large $k, \ell+1<$ $k+C^{\prime} \log k$, for any $C^{\prime}>C$, and

$$
\begin{aligned}
0<1-\left(\eta_{k} / \xi_{k}\right) & \leq 1-\left(\xi_{\ell+1} / \xi_{k}\right) \\
& =1-(K(\ell+1) / K(k)) \\
& <1-\left(K\left(k+C^{\prime} \log k\right) / K(k)\right) .
\end{aligned}
$$

But $(K(\theta k) / K(k))<\varepsilon$ if $\theta$ is suitably chosen, and the result follows.

As we mentioned, under the generality of our assumptions, we cannot expect a true second asymptotic term. Indeed, we have obtained only a nontrivial lower bound for $\eta_{k} / \xi_{k}$. Nevertheless, as crude as our method is, it seems to produce an error of the correct order of magnitude. For example, for $F(x)=\sin x$, it is known that

$$
F_{k}(a)=(3 / k)^{1 / 2}\left\{1-\frac{3}{10} \frac{\log k}{k}+o\left(\frac{\log k}{k}\right)\right\},
$$

(see [1]) if $a$ is small. If we set $G(x)=B x^{n}(1+0(x))$ and assume that $0<$ $G(y)-G(x)<y-x$ for $0<x<y<A$, and we also assume for simplicity that $n>2$, then a simple calculation gives $K(x)=(B(n-1) x)^{1 / 1-n}\{1+0(1 / x)\}$. It follows that $Q$ satisfies the hypothesis of Theorem 1 . Hence,

$$
(B(n-1) k)^{1 / 1-n}>\eta_{k}>(B(n-1) k)^{1 / 1-n}\{1-D(\log k / k)\}
$$

for suitable $D$.

Finally, for $G(x)=x^{2} \exp (-1 / x)$, another routine computation gives

$$
\frac{1}{\log k}>\eta_{k}>\frac{1}{\log k}\left\{1-\frac{C}{k}\right\} \text {. }
$$

\section{REFERENCES}

1. N. G. de Bruijn, Asymptotic methods in analysis, Wiley, New York, 1961.

2. V. Drobot and L. J. Wallen, Asymptotic iteration, Math. Mag. (to appear).

3. A. M. Ostrowski, Solutions of equations in Euclidean and Banach spaces, Academic Press, New York, 1973.

Department of Mathematics, University of Hawail at Manoa, Honolulu, Hawail 96822 